\title{
NETWORK MODEL FOR THERMAL CONVERSION OF HETEROGENEOUS BIOMASS GRANULES
}

\author{
Laura Leja ${ }^{1}$, Uldis Strautins ${ }^{2}$ \\ ${ }^{1}$ University of Latvia, Latvia; \\ ${ }^{2}$ Institute of Mathematics and Computer Science, University of Latvia, Latvia \\ 1113172@edu.lu.lv, uldis.strautins@lu.lv
}

\begin{abstract}
The focus of this research is on the development of a network model for efficient simulation of the thermal conversion of heterogeneous biomass granules during gasification or combustion processes. The network model for heat and mass transport in a porous medium is constructed, which treats heterogeneous constituents of granules (such as wood and straw) as nodes and common surfaces as edges connect them and allow heat and mass to be transported. Reactions with Arrhenius kinetics are used to model the thermal decomposition of biomass. The gas is assumed to be an incompressible ideal gas flowing through a porous medium. A pressure correction scheme is used to model the mass transfer. Conductive and convective heat transfer equations with source terms are used for temperature modeling. Examples in 2D and 3D are presented. This network model can be used as a module in more complex biomass conversion models, such as the Lagrangian particle model in combination with the Eulerian model for fluid dynamics. Improved understanding of these complex processes contributes to enhancing the use of renewable resources, such as wood, straw, peat, for a variety of industry and household applications, leading to more carbon - neutral economy, as well as greater use of local resources.
\end{abstract}

Keywords: network model, thermal conduction, graph, heterogeneous biomass granules.

\section{Introduction}

Increasing the preconditions for sustainable energy is the basis for looking for ways to use renewable energy sources, such as the efficient use of biomass granules as an alternative to fossil fuels and as an important raw material for renewable energy production. Biomass is also a carbon dioxide $\left(\mathrm{CO}_{2}\right)$ neutral fuel and therefore cannot affect the increase in emissions to the environment [1]. The European Union has set binding energy policy targets for 2030, which require not only a $40 \%$ reduction in greenhouse gas emissions, but at least $32 \%$ of energy consumption must come from renewable sources and an increase in energy efficiency of at least $32.5 \%$ for smart, sustainable and comprehensive development [2].

Emphasis is placed on thermal conversion of heterogeneous granules, as it allows the use of different types of biomass and agricultural waste and forestry raw materials, which ensures more profitable and efficient production, thus creating the ecological attractiveness of fuels. The use of heterogeneous biomass granules for residential heating is one of the ways to optimize the use of local bioresources while reducing dependence on fossil fuels and thus reducing overall emission of greenhouse gasses.

This study proposes a method to simulate the first stages of combustion of biomass granules composed, which consist of different materials, such as wood, peat and straw. We consider the thermal conversion of a heterogeneous granule, the geometry of which is described by a pore network. The aim is to study the physical processes (heat and mass transfer) that take place in a porous environment [3].

Thermal conversion research of heterogeneous biomass has been developed with different biomasses, e.g., when attention has been paid to the pyrolysis process [4], improved thermal transmission system gasification at high temperatures [5], for more complete combustion of mixed biomass leaving the least ash [6]. Similarly pore network models are and are being used in this way elsewhere, in oil refining [7], coal and charcoal combustion and gasification, but not in biomass, however, this type of model has not been applied in the literature to biomass to the best knowledge of the authors, but the same idea is expressed in one of the recent studies [8].

The combustion process itself is a complex process influenced by a wide range of conditions, such as exact composition of the biomass, the process characteristics of the thermal conversion (temperature, oxygen supply etc), also the observed quantities vary, e.g., environmental impact, volatile substances, etc. [9], therefore, it is difficult to accurately compare the experimental results with numerical simulations, especially if the biomass is a mixture of several materials. The proposed model predicts the conversion properties of mixed biomass granules depending on the composition. 


\section{Materials and methods}

The pore network model $[3,10]$ allows a wide flexibility of choice of geometry. We present only the simplest 2D case, which results in a structured rectangular network. Assume the heterogeneous granule is composed of three materials: straw, wood and peat. Divide the volume of the biomass into voxels of appropriate size and assume each voxel consists of a single material. A lattice is obtained, where each cell consists of its own material. Assume the network consists of $n \times m$ cells (Fig. 1), with biomass occupying the vertices where reactions take place, and heat and mass transfer happening through the edges that connect the vertices.

a)

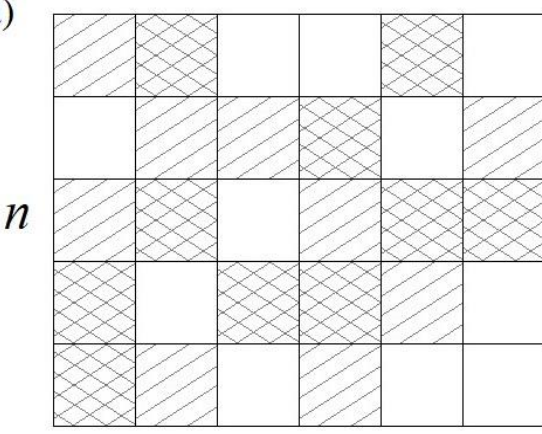

$m$ b)

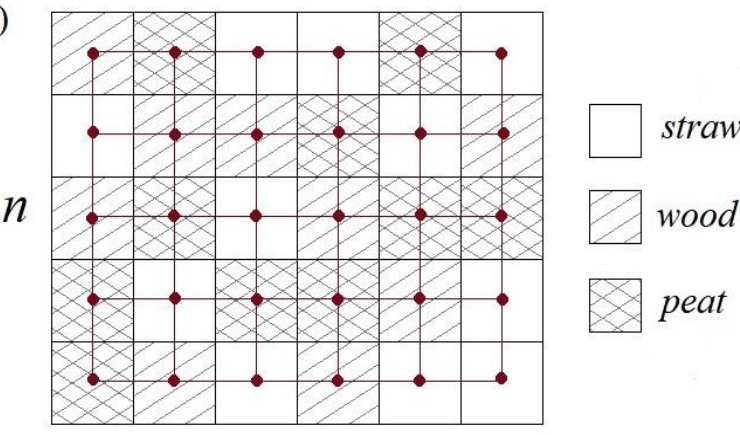

$m$

Fig. 1. Creating a network model for a heterogeneous granule; a) $\boldsymbol{n} \times \boldsymbol{m}$ size granule that consists of materials: wood, peat and straw; b) created network model for this granule

First order Arrhenius law is used to model the chemical reactions that govern the thermal decomposition of biomass:

$$
\frac{d M_{i}(t)}{d t}=-\alpha \exp \left(\frac{-E_{i}}{T_{i}(t)}\right)
$$

where $E_{i}$-activation energy that determines the rate of the reaction;

$M_{i}$ - remaining combustible material at $i-t h$ vertex at time $t$; $\alpha$ - kinetic constant of the reaction.

The heat produced is proportional to the loss of mass. When the reaction starts, volatile substances, moisture and heat are transported through the network [11].

For the created network model, each vertex is assigned a pressure $p_{j}$, which indicates gas pressure at the vertex $j$, but the edges are assigned flows $q_{i j}$, where the edge connects the vertices $i$ and $j$. The flows are scalar quantities on the directed edges. We assume incompressible flow with mass sources (the decomposition of biomass results in release of volatiles). Thus, in the network model, the sum of inflowing and outflowing mass of gas plus the released mass at each vertex has to be zero. This is the pressure correction method, which defines the pressure. The mass fluxes are corrected by adding to $q_{i j}$ the quantity

$$
q^{\prime}=\frac{p_{j}-p_{i}}{l_{j i}}
$$

where $l_{j i}$ - length of the edge connecting vertices $i$ and $j$.

The resulting condition is:

$$
\sum_{i i} Q_{i i}=0 .
$$

The corrected mass flux $Q$ is defined as $q_{i j}+q_{i j}^{\prime}=Q_{i j}$. The pressure correction equation is a system of linear equations with respect to $p_{j}$. It can be written down with the help of the Laplace operator

$$
\sum_{j \sim i} q_{j i}+\frac{p_{j}-p_{i}}{l_{j i}}=0 \Leftrightarrow \sum_{j \sim i} \frac{p_{i}-p_{j}}{l_{i j}}=\sum_{j \sim i} q_{j i} .
$$


In this case

$$
\sum_{j \sim i} \frac{p_{i}-p_{j}}{l_{i j}}
$$

is the Laplace operator of the graph. When the gas is heated, natural convection is induced.

Applying Darcy's law, we express the flow as follows:

$$
\vec{q}=k\left(\left(\rho\left(T_{0}\right)-\rho(T)\right) \vec{g}-\nabla p\right),
$$

where $k$-permeability coefficient divided by the dynamic viscosity of the gas.

The pressure $p$ does not contain a hydrostatic component and $\nabla p$ is the pressure gradient, but $\rho_{0}$ is air density in the ambient environment and $T_{0}$ is the ambient temperature. The mass balance equation is:

$$
\operatorname{div} \vec{q}=A,
$$

The term A denotes mass sources. We use the network analogue:

$$
A_{i} \approx k \sum_{j \sim i}-\left(\frac{p_{j}-p_{i}}{l_{i j}}+\left(\rho\left(T_{0}\right)-\rho_{e f f, j}\right) \vec{g} \cdot \vec{n}\right) \gamma_{j},
$$

where $\rho_{e f f, j}$ - effective density on the $j$ side, which can be calculated using upwinding;

$\gamma_{j}$ is the weight representing the cross-section of the link $i \sim j$;

$\left(\rho\left(T_{0}\right)-\rho_{e f f, j}\right) \vec{g} \cdot \vec{n}$ - buoyancy force $F_{A}$, what we express in terms of temperatures.

$$
\vec{g} \cdot \vec{n}\left(\rho\left(T_{0}\right)-\rho(T)\right) \approx a\left(1-\frac{T_{0}}{T}\right) .
$$

Mass transfer is coupled to heat transfer, which includes conduction, convection and heat sources. The conduction part is mathematically analogous to the pressure correction equation. The convection part is modelled as follows: we assume that the gas flowing through a vertex assumes its temperature. Thus, the temperature of a vertex is affected only by the adjacent edges, through which the gas flows into the vertex. The heat transfer from the gas to the vertex is proportional to the temperature difference, mass flow rate and heat capacity of the gas. Finally, the heat source is proportional to the rate of mass conversion at the vertex. This results in the heat transfer equations:

$$
c_{i} \rho_{i}\left(1-\phi_{i}\right) V_{i} \frac{d T_{i}}{d t}=\sum_{i \sim j} k_{i j} \frac{T_{j}-T_{i}}{l_{i j}} S_{i j}+\sum_{j \rightarrow i} \rho_{g, j} q_{j i}^{+} S_{i j} c_{p}\left(T_{j}-T_{i}\right)+Q_{i},
$$

where $c_{i}, \rho_{i}, \phi_{i}, V_{i}$ - heat capacity, density, porosity of biomass and total volume associated to $i$ the vertex,

$k_{i j}, S_{i j}, q_{j i}^{+}-$conduction coefficient, effective cross section and volume flux from $j$ to $i$ (set to zero if negative),

$c_{p}$ - heat capacity of the gas;

$Q_{i}$ - heat source it is

$$
Q_{i}=\lambda m_{i} e^{\frac{E}{R(T)}},
$$

where $\lambda$-heat release coefficient;

$m_{i}$ - mass;

$\frac{E}{R(T)}-$ reaction constant.

The equations are supplemented by initial conditions and suitable boundary conditions for pressure and temperature. Homogeneous Neumann boundary conditions for pressure describe impermeable walls; Dirichlet pressure boundary conditions describe parts of boundary which allow mass exchange.

This approach allows using spatially and temporarily varying material coefficients such as porosity, permeability, heat conductivity, heat capacity, dynamic viscosity of gas, and even anisotropic conductivities, and hence this model is suitable for simulating intra-particle and inter-particle thermal conversion processes for heterogeneous biomass materials. 
The 3D Model is based on the principle of a 2D model, as it retains both the vertices and the corresponding edges and neighbours, but also adds the coefficients responsible for the composition: cellulose, hemicellulose and lignin. The transfer to 3D and more complex geometries is straightforward.

\section{Results and discussion}

Two types of mathematical models were compared: the first heterogeneous pellet model (straw, wood, peat) the second homogeneous pellet model consists exclusively of wood, peat or straw. The mixed distribution of biomass is formed randomly with a percentage distribution: $40 \%$ of the granules are made of straw, $20 \%$ are made of wood shavings and the remaining $40 \%$ are made of peat. Unused energy is taken as a measure, which is interpreted as the unburned mass in the network model. If the granules are homogeneous, that is, the activation energy is the same everywhere, no multiple realization are required, because no change occurs and all simulations are the same, but if the geometry of the network model is randomly arranged with different materials, then it is necessary to look at several realizations.

If the coefficient is changed in the heat conduction equation, which determines how much heat is released in one time interval, then significant differences can be noticed in the results. The temperature must be chosen appropriately, because if the temperature is high enough, the maximum reaction rate is reached and the results remain constant. As well as the Arrhenius function may reflect invalid data and the activation energy $E_{i}$ becomes insensitive to change. If the granules are homogeneous, that is, the activation energy is the same everywhere, no multiple realization is required, because no change occurs and all simulations are the same, but if the geometry of the network model is randomly arranged with different materials, therefore, it was necessary to look at several implementations with mass distribution and with a fixed time the same for both models.

a)

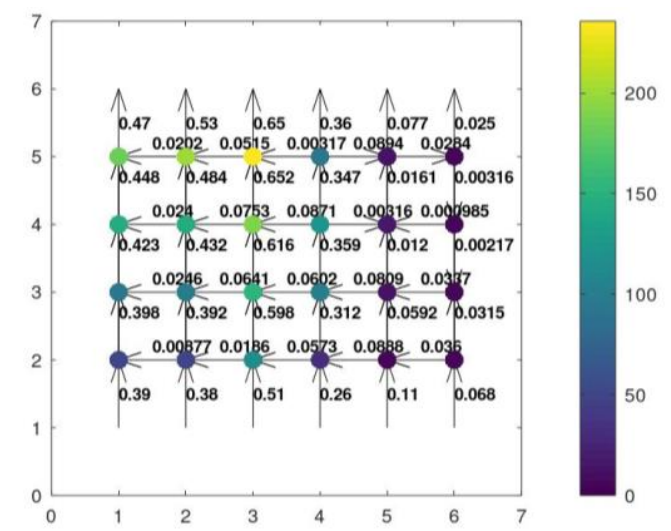

b)

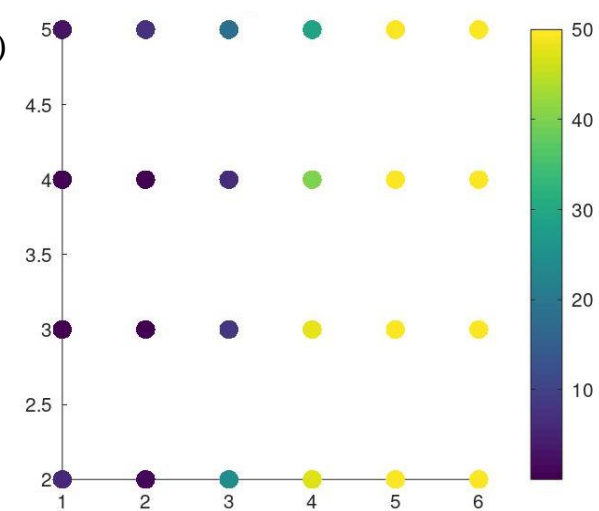

Fig. 2. 2D realization of heterogeneous model: $a$ - temperature and flow velocity; $b$ - remaining mass of fuel at final time; the sum of unburned mass is 640.06 (dimensionless units)

a)

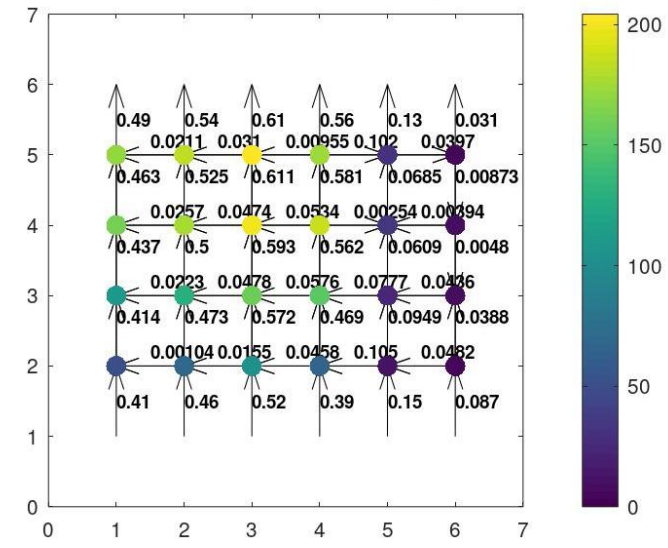

b)

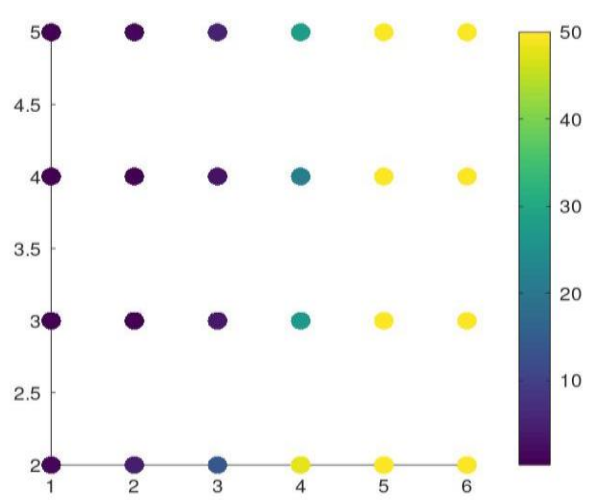

Fig. 3. 2D realization of a homogeneous (wood) model: a - temperature and flow velocity; $\mathrm{b}$ - remaining mass of fuel at final time; the sum of unburned mass is 561.57 (dimensionless units) 

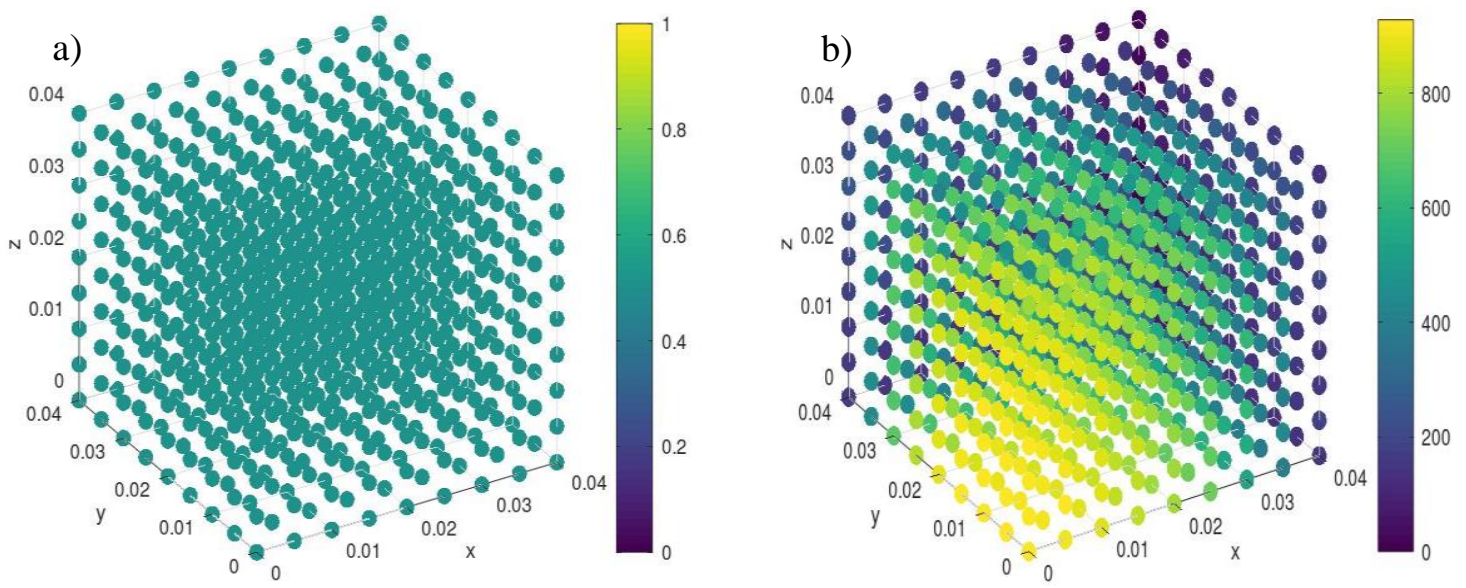

Fig. 4. 3D realization of a biomass (straw) model: $a$ - temperature; $b$ - remaining mass of hemicellulose at final time
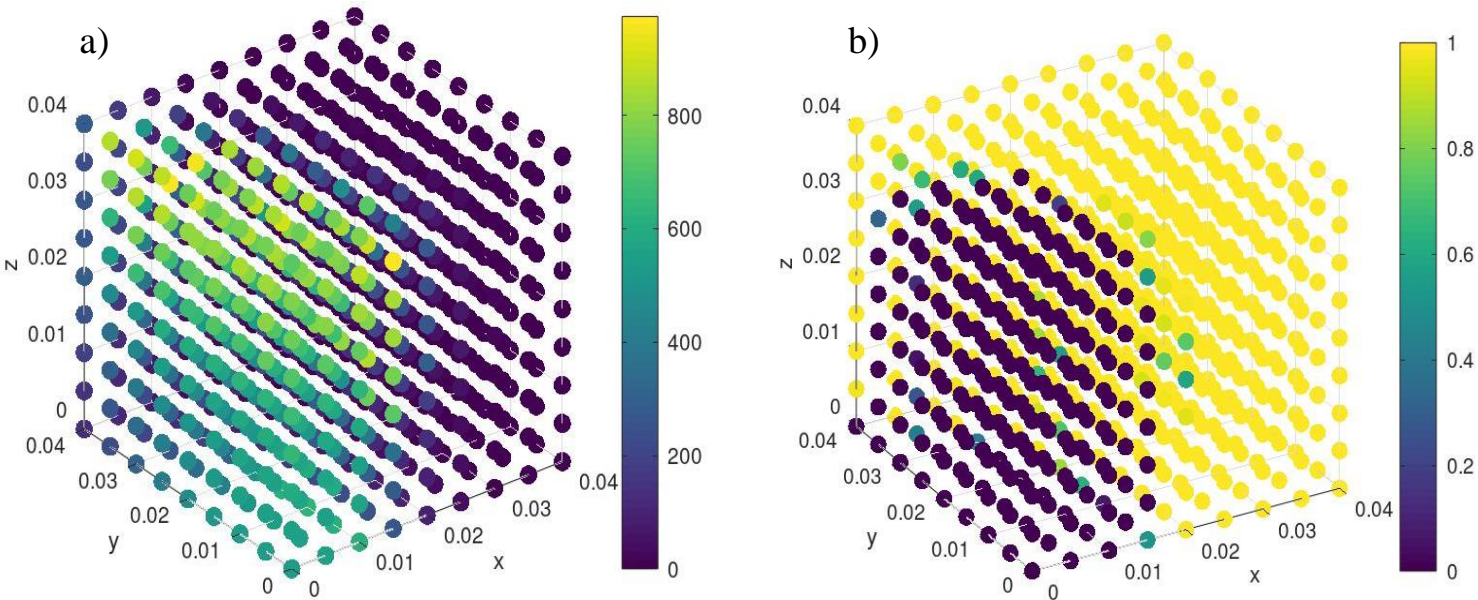

Fig. 5. 3D realization of a biomass (wood) model: $a$ - temperature; $b$ - remaining mass of hemicellulose at final time
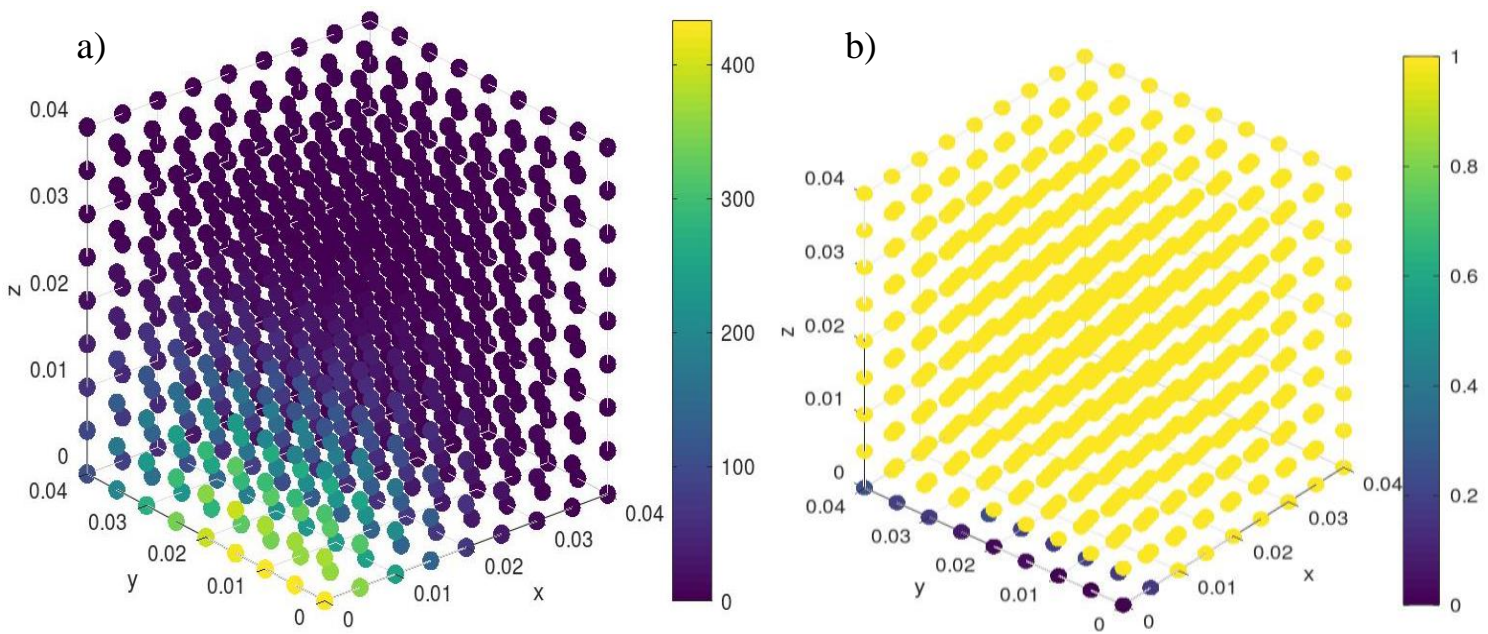

Fig. 6. 3D realization of a biomass (peat) model: $a$ - temperature; $b$ - remaining mass of hemicellulose at final time 
For both models (heterogeneous and homogeneous) we assume the same initial conditions and in the time interval from 0 to 1 (assumed to be a dimensionless quantity) and a time step of 0.001 and ignite the very first vertex. In both figures (Fig. 2, 3), the first illustrations (a) show the temperature (coloured) and the flow rate (numbers and direction), but $b$ is the mass remaining at the last time step was chosen for both models. In the visualization of the simulation (Fig. 2) in the case of the heterogeneous model the unburned mass has remained more than the homogeneous (wood) model (Fig. 3). Which could indicate that in the specific realization the temperature of peat as a material has not reached a sufficiently high temperature, therefore the unburned mass remains more.

Looking at all the heat generated, which shows that a certain amount of mass has been burned, it is more possible to determine how much heat is generated by each unit of mass distributed. But in order to be able to more objectively evaluate the heterogeneous and homogeneous model in the initial conditions we consider three different heat release coefficients $(\lambda)$, namely 10,40 and 50 . If the heat conduction of the equation assumes that the heat release coefficient is 10 , which is relatively small, then more unburned material will remain. The results show that the heterogeneous average unburned mass of granules (111.23; the unit of mass is taken as dimensionless units) remains more than that of a homogeneous pellet with straw (1057.4), but less than that of a homogeneous pellet formed separately from wood (1130.2) or peat (1161.6). The higher the activation energy, the higher the amount of heat required to be able to burn the mass of the substance.

If in the heat conduction equation the coefficient is assumed to be 4 times higher than in the previous implementations, then it is observed that the mass burns much better, as it should be, because a larger amount of heat is released. For heterogeneous granules, the average residual mass (701.7) is lower than for pellets formed homogeneously from straw (742.43) or peat (708.96), but higher for granules formed from wood (688.42). But if we assume a coefficient of 50, then with the conclusion that heterogeneous granules burn better and leave less unused energy, mass (531.6), but for homogeneous straw (636.76), wood (561.57) and the mass of peat (579.11), not so well burned and more unused energy left over. Comparing the results of this study with obtained data with similar experimental results available in the literature [12]. The results show that straw ignites faster, so straw has the highest heat release at lower temperatures. For both wood and peat a higher temperature is needed to be able to burn, but according to the data, when wood burns, heat release occurs more the loss of mass, because with increasing temperature, it reaches several peaks, which indicates a higher heat output.

The 3D Variable parameters - different for each material (lignin, cellulose and hemicellulose) - heat production coefficient, porosity coefficient, Arrhenius kinetic constant, are functions of the respective biomass material, all these parameters affect the thermal decomposition of biomass cellulose, hemicellulose, lignin. Biomass - straw (Fig. 4), the reaction took place rapidly, the mass is burnt (between 0.4 and 0.6), it is left over and leaves behind a lot of ash and unburned particles.3D Model with wood biomass (Fig. 5), the reaction took place rapidly, because the mass is well burned (between 0.4 and 0.6). Model with peat (Fig. 6), biomass ignition is much slower, the burnt mass (0-0.4), the remaining mass is relatively large, which is not affected by the flame at all, against burnt mass.

\section{Conclusions}

The numerical implementation of the model proposed for such a porous material makes it possible to represent this complex combustion process simply and with low computational costs. Numerical results show that for homogeneous pellets the loss of mass indicates that the weight of straw decreases more rapidly than for wood and finally for peat, but for heterogeneous pellets consisting of different biomasses, the weight loss dependence is no longer so easy to determine. Existing straw in these heterogeneous granules may behave differently due to lack of oxygen and other conditions. It is planned to develop and improve this model by adding a dependence on the moisture and composition of substances in biomass, so that it is even more similar to the real situation in the furnace. The modelling procedures developed for this application will be transferred to modelling of other physical processes. These granules set model will be immersed in a larger model that will look at the air - filled space around the granules, where the combustion process or biogas collection in the balloon can take place, for further modelling of the gas dynamics equations. 


\section{References}

[1] Vasilev S. V., Baxter D., Andersen L.K., Vasileva C.G., Morga T.J. An overview of the organic and inorganic phase composition of biomass. Fuel, vol. 94, 2012, pp. 1-33.

[2] 2030 climate \& energy framework, Policy, [online] [28.02.2021]. Available at: https://ec.europa.eu/clima/policies/strategies/2030_en.

[3] Xiong Q., Baychev T.G., Jivkov A.P. Review of pore network modelling of porous media: Experimental characterisations, network constructions and applications to reactive transport. Journal of Contaminant Hydrology, 192, 2016, pp. 101-117.

[4] Larina O. M., Sinelshchikov V. A., Sytchev G. A. Comparison of thermal conversion methods of different biomass types into gaseous fuel. Journal of Physics: Conference Series, 2016, 774, 012137, DOI:10.1088/1742-6596/774/1/012137.

[5] Stasiek J., Szkodo M. Thermochemical Conversion of Biomass and Municipal Waste into Useful Energy Using Advanced HiTAG/HiTSG Technology. Energies, 2020, 13, 4218, DOI:10.3390/en13164218.

[6] Barmina I., Valdmanis R., Zake M., Ozola L., Strautins U. Development of gasification/combustion characteristics at thermo-chemical conversion of biomass mixtures. Engineering for Rural Development, 2017, pp. 54-59.

[7] Su J., Wang L., Gu Z., Zhang Y., Chen C. Advances in Pore-Scale simulation of Oil Reservoirs. Energies, 2018, 11, 1132; DOI:10.3390/en11051132.

[8] Fatehi H., Weng W., Li Z., Bai X., Alden M..Recent Development in Numerical Simulations and Experimental Studies of Biomass Thermochemical Conversion. Energy fuels, 2021, DOI: 10.1021/acs.energyfules.0c04139.

[9] Di Blasi C. Modeling chemical and physical processes of wood and biomass pyrolysis. Progress in Energy and Combustion Science, 2008, vol. 34, Issue 1, pp. 47-90.

[10] Rabbani A., Babaei M., Javadpour F. A Triple Pore Network Model (T-PNM) for Gas Flow Simulation in Fractured, Micro-porous and Meso-porous Media, 2020, 132, pp. 707-740.

[11] Chen Y., Yang J., Zhang Y., Liu K., Liang S., Xu X., Hu J., Yao H., Xiao B. Kinetic simulation and prediction of pyrolysis process for non-metallic fraction of waste printed circuit boards by discrete distributed activation energy model compared with isoconversional method. Environmental Science and Pollution Research, 2018, pp. 3636-3646.

[12] Barmina I., Kolmickovs A., Valdmanis R., Vostrikovs S., Zake M. Thermo-Chemical Conversion of Microwave Activated Biomass Mixtures. VIII International Scientific Colloquium on Modelling for Materials Processing. IOP Conf. Series: Materials Science and Engineering. 355, 2018. 